\title{
Fuel-Operated Auxiliary Heaters Are a Major Additional Source of Vehicular Particulate Emissions in Cold Regions
}

\author{
Panu Karjalainen ${ }^{1}{ }^{\mathbb{D}}$, Markus Nikka ${ }^{2}$, Miska Olin ${ }^{1} \mathbb{D}$, Sampsa Martikainen ${ }^{1} \mathbb{D}$, Antti Rostedt ${ }^{1}$, Anssi Arffman ${ }^{2} \mathbb{D}$ \\ and Santtu Mikkonen $3,4, * \mathbb{D}$ \\ 1 Aerosol Physics Laboratory, Tampere University, P.O. Box 692, 33014 Tampere, Finland; \\ panu.karjalainen@tuni.fi (P.K.); miska.olin@tuni.fi (M.O.); sampsa.martikainen@tuni.fi (S.M.); \\ antti.rostedt@tuni.fi (A.R.) \\ 2 Dekati Ltd., Tykkitie 1, 36240 Kangasala, Finland; markus.nikka@dekati.fi (M.N.); \\ anssi.arffman@dekati.fi (A.A.) \\ 3 Department of Applied Physics, University of Eastern Finland, P.O. Box 1627, 70211 Kuopio, Finland \\ 4 Department of Environmental and Biological Sciences, University of Eastern Finland, P.O. Box 1627, \\ 70211 Kuopio, Finland \\ * Correspondence: Santtu.mikkonen@uef.fi
}

\section{check for} updates

Citation: Karjalainen, P.; Nikka, M.; Olin, M.; Martikainen, S.; Rostedt, A.; Arffman, A.; Mikkonen, S.

Fuel-Operated Auxiliary Heaters Are

a Major Additional Source of

Vehicular Particulate Emissions in Cold Regions. Atmosphere 2021, 12, 1105. https://doi.org/10.3390/ atmos12091105

Academic Editors: Salah Khardi and Nathalie Bernoud-Hubac

Received: 21 July 2021

Accepted: 23 August 2021

Published: 26 August 2021

Publisher's Note: MDPI stays neutral with regard to jurisdictional claims in published maps and institutional affiliations.

Copyright: (c) 2021 by the authors. Licensee MDPI, Basel, Switzerland. This article is an open access article distributed under the terms and conditions of the Creative Commons Attribution (CC BY) license (https:/ / creativecommons.org/licenses/by/ $4.0 /)$.

\begin{abstract}
Fuel-operated auxiliary heaters (AHs) can be notable sources of particle emissions from vehicles. The emissions of AHs are unregulated, and the number of devices is high; therefore, they make considerable contributions to local air quality, and even the global emissions budget. Experiments for studying the emissions were performed in Finland for a total of eight selected vehicles with Original Equipment Manufacturer (OEM) AHs installed, including both diesel- and gasoline-operated heaters. We present the numerical results of particle emissions and compare the particle concentrations in the $\mathrm{AH}$ exhaust to values found in the tailpipe exhaust of the same vehicle. Our results show that the emissions from auxiliary heaters are typically several orders of magnitude higher than of a car exhaust when idling. This raises the question of whether the use of heaters is justified based on the goal to reduce total emissions from vehicle use; furthermore, whether fuel-operated heaters should also be applied in electric vehicles for cabin heating. More research will be needed to characterize the emissions more thoroughly to understand the air quality and climate effects from AHs, and to provide further recommendations on the use of these heaters.
\end{abstract}

Keywords: vehicle emissions; particulate emissions; auxiliary heaters; particle number

\section{Introduction}

Two major human-induced environmental challenges are poor air quality and the acceleration of climate change. The emissions originating from traffic are a significant factor in both. Exposure to respirable particles in the air, meaning particulate matter below 2.5 micrometers in size (PM2.5), is an immense global challenge estimated to cause approximately 8.9 million premature deaths per year worldwide [1]. In addition to severe health problems, aerosols are an important anthropogenic component affecting global warming by contributing to Earth's radiation balance with high uncertainties. In an urban environment, traffic exhaust fumes are one of the most significant sources of aerosol particles and condensable gases affecting new particle formation in the air. The main components of traffic-originated particles are black carbon (BC) and primary and secondary organic aerosols. The fractions and size distributions of particles vary greatly between emission sources and ageing state. Combustion-originated particles can transport long distances and affect, e.g., cloud formation and surface albedo, especially on snowy and icy surfaces.

Due to the adverse effects, the emissions of vehicles are regulated. Legislation constantly brings more stringent emission limits, measuring the most conventional aerosol 
quantity, particulate mass. In some cases, mass emissions have been reduced to below the detection limit. However, mass may not be the best measure for adverse health effects [2]; therefore, updates in regulatory particle emission limits have introduced a particulate number (PN) limit. In Europe, PN emission levels of non-volatile particles for light-duty (LD) diesel vehicles have been implemented since 2009 (Euro $5 \mathrm{~b}$ emission standards). Additionally, starting from Euro 6 emission standards implemented in 2014, the non-volatile exhaust PN has been regulated for gasoline direct injection (GDI) passenger cars. This non-volatile particle regulation currently includes particles greater than $23 \mathrm{~nm}$ in size. The PN limit for diesel passenger cars has de facto enforced the use of diesel particulate filters (DPFs) in the exhaust line. These filters have been shown to be very efficient in PN reduction, e.g., a reduction efficiency of up to $99.998 \%$ has been reported [3].

When a vehicle is started after a long parking time under sub-zero conditions or close to them, heating of the engine, vehicle body and cabin is needed. This need originates from various aspects such as the prevention of engine wear right after a cold start, melting accumulated ice from the vehicle windows, warming up the ventilation air to reduce fogging of the windshield, and increasing the comfortability for passengers. Typical strategies to heat up the engine include starting the engine and holding it on idle for several minutes, or using an external heating source before engine start-up. The latter is often intended to reduce engine wear in very cold conditions and to make sure that the engine starts. External heaters can be electrically operated-having a drawback of the requirement of the power grid connection — or they can be fuel-burning auxiliary heaters (AHs), providing higher power output and more freedom for selection of the parking location. Fuel-operated AHs often also activate during normal operation of the vehicle after a cold start to increase the heating rate of the engine and cabin. In some cases, manual usage of the heater is impossible; instead, it automatically activates during the vehicle start under cold conditions. Fuel-operated AHs use the same fuel tank as the internal combustion engine (ICE), but they have a separate exhaust pipe, right below the heating unit at the front of the vehicle.

Unlike in the case of vehicle exhausts, $\mathrm{AH}$ exhaust emissions are not regulated because it is an additional heat source, not the source of mechanical energy. Thus, fuel-operated AHs can be a surprisingly large source of particle emissions that cannot easily be distinguished in air quality measurements in traffic environments. The total number of AHs globally is unavailable, but the total sales of two major manufacturers in this subdivision exceed EUR one billion annually [4,5]. Thus, the number of devices sold annually is high. To the best of our knowledge, the aerosol emissions from fuel-operated auxiliary heaters have not been studied and reported in the scientific literature. Previous studies have only focused on improving the thermal efficiency or fuel economy of the heaters [6]. Conversely, AHs have also been used as artificial soot generators in laboratories after modifying their combustion parameters [7], which do not represent normal use emissions.

In this paper, we present evidence that AHs can be significant sources of particle emissions. Experiments were performed in Finland for a total of eight selected vehicles with installed OEM AHs. Both diesel and gasoline technologies were covered. We present numerical results of particle emissions and compare the particle concentrations in the $\mathrm{AH}$ exhaust to the values found in the tailpipe exhaust of the same vehicle.

\section{Materials and Methods}

\subsection{Vehicles, Fuels, Lubricants}

A fleet of eight light-duty vehicles, registered in Finland and conforming to European emission regulations, were obtained from daily use for the experiments (Table 1). This small vehicle fleet included medium and large passenger cars, space wagons and a van. All vehicles were equipped with an AH combusting the same fuel as the ICE. Four of the vehicles ran on gasoline and four ran on diesel. Commercial-pump-grade fuels typical in Finland were used: gasoline is 95 octane fuel (95E10 standard), where ethanol is blended up to $10 \mathrm{vol} \%$, whereas diesel has a renewable fuel fraction of at least 7\% (EN590 standard). 
The vehicles had been operating for between 0 and 19 years on the road. We know that the AH in the oldest vehicle, a BMW 530d, had been replaced at some point with a used spare part of the same make and model as the original unit, but otherwise, to the best of our knowledge, all the vehicles were using their factory-installed AHs. To provide some perspective of the AH activity, a Seat Alhambra, with a mileage of 168,000 km, had operated the flame of the $\mathrm{AH}$ for $1250 \mathrm{~h}$ during its lifetime. This corresponds up to $40 \%$ of the total usage of the vehicle when estimating it from the mileage of the vehicle and the approximation of a typical average driving speed of $60 \mathrm{~km} / \mathrm{h}$. Most of this is probably passive use where the driver does not activate the AH manually. Instead, the engine control automatically activates the $\mathrm{AH}$ when the ambient temperature is below the threshold value.

\subsection{Driving Conditions, Preconditioning, Test Procedure}

The vehicles were tested in a garage at the premises of Dekati Ltd. (Kangasala, Finland) with the following test protocol. The vehicle was driven into the garage and left on idle. The heated sampling line was connected to the vehicle tailpipe, and the exhaust concentrations on idle (low idle) were measured with real-time particle instrumentation. In this way, the protocol was similar to that which will be performed in upcoming annual periodical testing and inspection (PTI) emission tests for vehicles, e.g., in The Netherlands [8]. Notably, although a Skoda Octavia 1.0 used hybrid technology, it had no effect on the emissions on idle because the Mild Hybrid Electric Vehicles (MHEV) technology used always operates the main engine on idle. After idle tests, the heated sampling line was connected to the exhaust pipe of the $\mathrm{AH}$, located under the engine for all the tested vehicles. The AHs were activated with a manual start button, and after selected time, they were manually shut down.

Table 1. Tested AH-containing vehicles with details. MHEV denotes mild hybrid electric vehicle, which uses an electric motor only for assisting the main engine and does not typically run alone.

\begin{tabular}{|c|c|c|c|c|c|c|c|}
\hline Vehicle & Registration Year & Emission Class & Mileage (km) & Fuel & Engine Size (L) & Power (kW) & Additional Information \\
\hline $\begin{array}{c}\text { Skoda } \\
\text { Octavia } 1.0\end{array}$ & 2020 & Euro 6 & 1000 & Gasoline & 1.0 & 81 & MHEV \\
\hline $\begin{array}{c}\text { Skoda } \\
\text { Octavia } 2.0\end{array}$ & 2019 & Euro 6 & 21,000 & Gasoline & 2.0 & 140 & \\
\hline VW Golf 1.0 & 2019 & Euro 6 & 49,000 & Gasoline & 1.0 & 85 & \\
\hline VW Golf 1.4 & 2015 & Euro 5 & 100,000 & Gasoline & 1.4 & 90 & \\
\hline MB Vito & 2015 & Euro 6 & 59,000 & Diesel & 2.1 & 100 & DPF \\
\hline $\begin{array}{c}\text { Seat } \\
\text { Alhambra }\end{array}$ & 2012 & Euro 5 & 168,000 & Diesel & 2.0 & 103 & DPF \\
\hline VW Sharan & 2011 & Euro 5 & 164,000 & Diesel & 2.0 & 103 & DPF \\
\hline BMW 530d & 2002 & Euro 3 & 375,000 & Diesel & 3.0 & 142 & $\begin{array}{l}\text { Possibly altered } \\
\text { aftertreatment }\end{array}$ \\
\hline
\end{tabular}

\subsection{Aerosol Sampling and Dilution}

The exhaust particle concentrations were measured with state-of-the-art sampling and instrumentation setup, as shown in Figure 1. Raw exhaust emissions were measured undiluted with the combination of a sampling unit and a ePNC ${ }^{\mathrm{TM}}$ (Dekati Ltd. Finland) [9] electrical particle sensor. The sampling unit was a protype of a commercial version designed for annual automotive PTI at PTI stations. It was composed of a volatile particle evaporation tube operating at $300{ }^{\circ} \mathrm{C}$ and a critical orifice assembled after the evaporation chamber to reduce the pressure from ambient to 400 mbars (absolute). The sampling unit was followed by the ePNC ${ }^{\mathrm{TM}}$ that operated in reduced pressure conditions to inhibit moisture condensation and particle formation inside the sensor.

For other instrumentation, exhaust gas was diluted with the eDiluter Pro ${ }^{\mathrm{TM}}$ (Dekati Ltd. Finland) [9] in order to obtain concentrations and temperatures suitable for the instruments. Raw exhaust emissions were sampled to the diluter with a heated sampling line kept at $200{ }^{\circ} \mathrm{C}$. In the eDiluter Pro ${ }^{\mathrm{TM}}$, the exhaust was first hot-diluted with $300{ }^{\circ} \mathrm{C}$ filtered compressed air, and secondary dilution was performed with room temperature compressed 
air. The primary and secondary dilution ratios were varied between 5 and 15 , and the total dilution ratio varied between 75 and 225 .

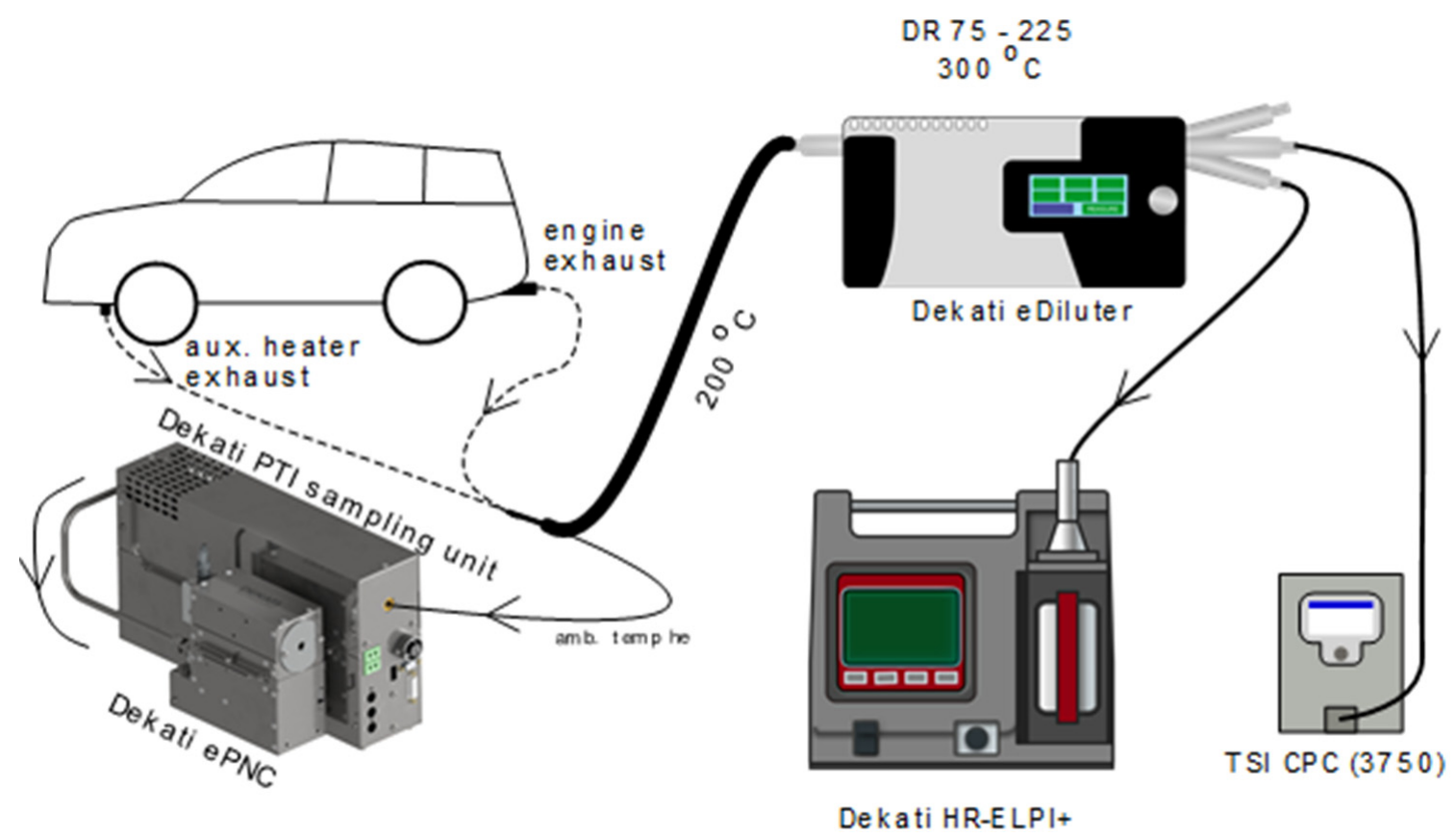

Figure 1. Experimental setup with sampling locations (aux-heater exhaust pipe and engine exhaust pipe), sampling parameters and aerosol instrumentation.

\subsection{Aerosol Instrumentation}

The ePNC, which measured the undiluted exhaust, is a new electrical detection-based particle number counter built to fulfill the requirements of the upcoming New Periodic Testing and Inspection (NPTI; [10]) of vehicle regulations for particle number counts. More detailed information on the ePNC can be found in Arffman et al. (2021) and Dekati Ltd. $(2021)[9,11]$. Briefly, particles are first charged with a corona charger (excess ions are removed with an annular trap), and subsequently, a fraction of the particles is collected in a channel-type diffusion collector. From the diffusion collector, the electrical current induced by the collected charged particles is measured with a low-noise electrometer. The amplified electrometer signal is almost directly proportional to the particle number count in the size range of 20-200 $\mathrm{nm}$ because the diffusion collection efficiency decays with the increasing particle size compensating the increase in the particle charging probability. The charger and the diffusion collector operate in reduced pressure conditions that slightly reshape the charging probability and the diffusion collection efficiency, optimizing the instrument response curve shape to meet the NPTI criteria [8]. The reference instruments for the ePNC were a condensation particle counter (CPC, model 3750, TSI Inc. Shoreview, MN, USA) and an electrical low-pressure impactor (HR-ELPI+, Dekati Ltd. Finland [9,12]), measuring the diluted and dried soot particles. The CPC measured the number concentration, and the HR-ELPI+ measured the particle number size distribution.

\section{Results and Discussion}

The exhaust particle concentrations of an AH vary over time depending on the phase of the combustion cycle. In Figure 2, time series of examples of particle number concentrations measured from the $\mathrm{AH}$ exhaust during operation of the heater, as well as from the vehicle exhaust during engine idling as comparison, are presented. The results obtained with the CPC, ePNC and HR-ELPI+ are shown. The upper subfigure is an example of a dieseloperated vehicle and heater (Seat Alhambra), whereas the lower subfigure represents a gasoline vehicle (Skoda Octavia). The AH time series includes the startup and turnoff 
of the device, whereas the vehicle engine time series are stably idling throughout. We observed some variability between the operations of different heaters, but the typical particle concentration profile during heater operation followed a pattern: the startup caused an initial spike of high concentration, after which it eventually reached a plateau stage. It should be noted that different heaters operate differently during the turnoff phase. Some of the heaters used in the tests began a cooldown sequence after receiving the shutdown command, during which the particle concentration of the exhaust was elevated. For both vehicles, we can see that the levels of AH exhaust numerical concentrations are somewhat four orders of magnitude larger than in the idling exhaust, but dependent on the $\mathrm{AH}$ operation cycle. During the cycle, the variation in AH exhaust concentrations is 1-2 orders of magnitude, reaching levels higher than $10^{7} \# / \mathrm{cm}^{3}$, and decreasing to $10^{5} \# / \mathrm{cm}^{3}$ with the diesel heater in the plateau. The blue areas indicate the time periods of the plateau, from which the results for the comparison presented in Figure 3 were calculated.
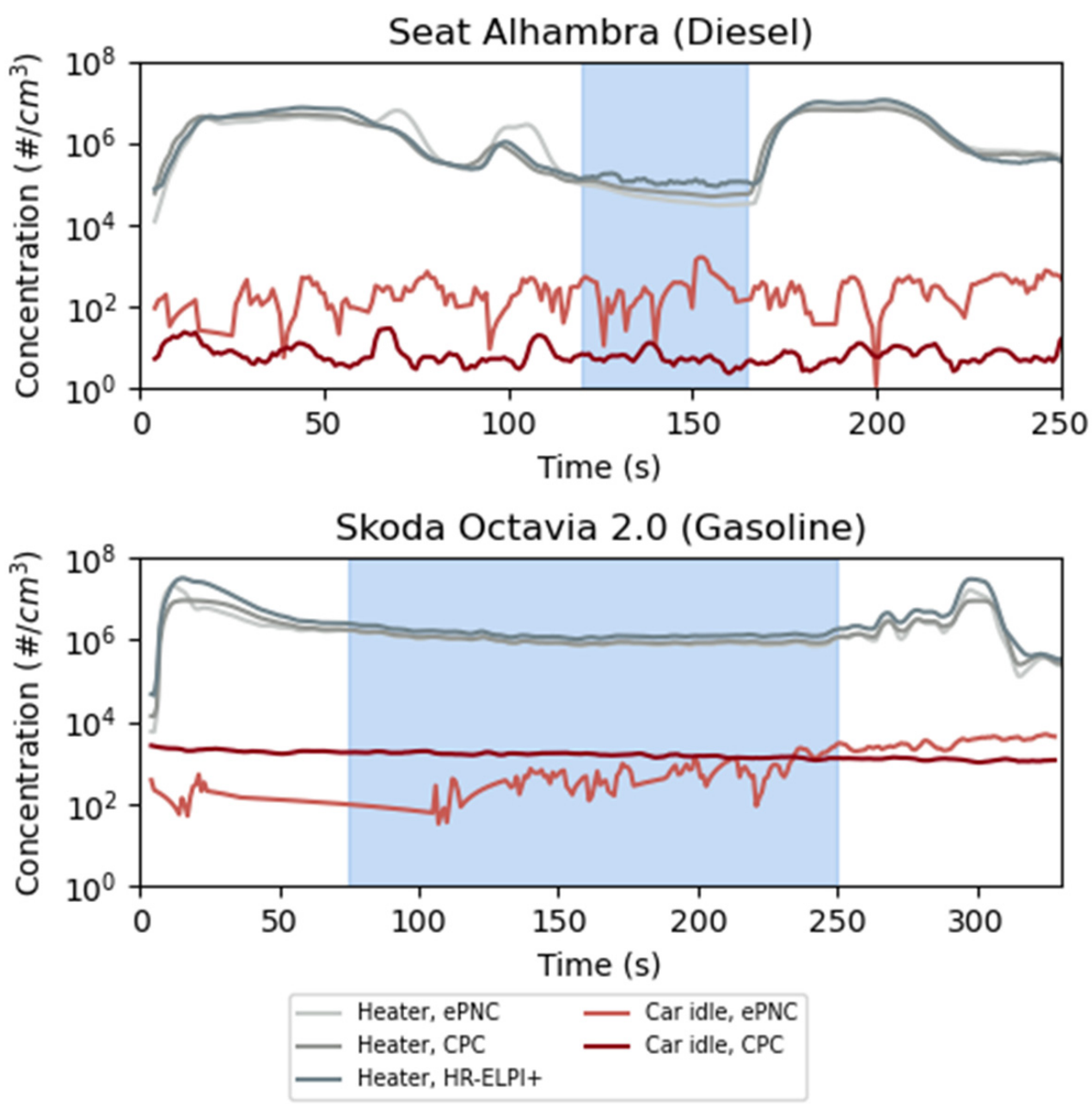

Figure 2. Time-series of particle number concentrations during auxiliary heater operation. Particle number concentrations during vehicle engine idling are also presented, to give perspectives of the magnitude of $\mathrm{AH}$ emissions. The blue background indicates the period of the plateau where the emission was fairly stable, and from which the results for the comparison presented in Figure 3 were calculated. During the idle measurement, the particle number concentration was close to the detection limit of the ePNC, causing large variation between the ePNC and the CPC. 


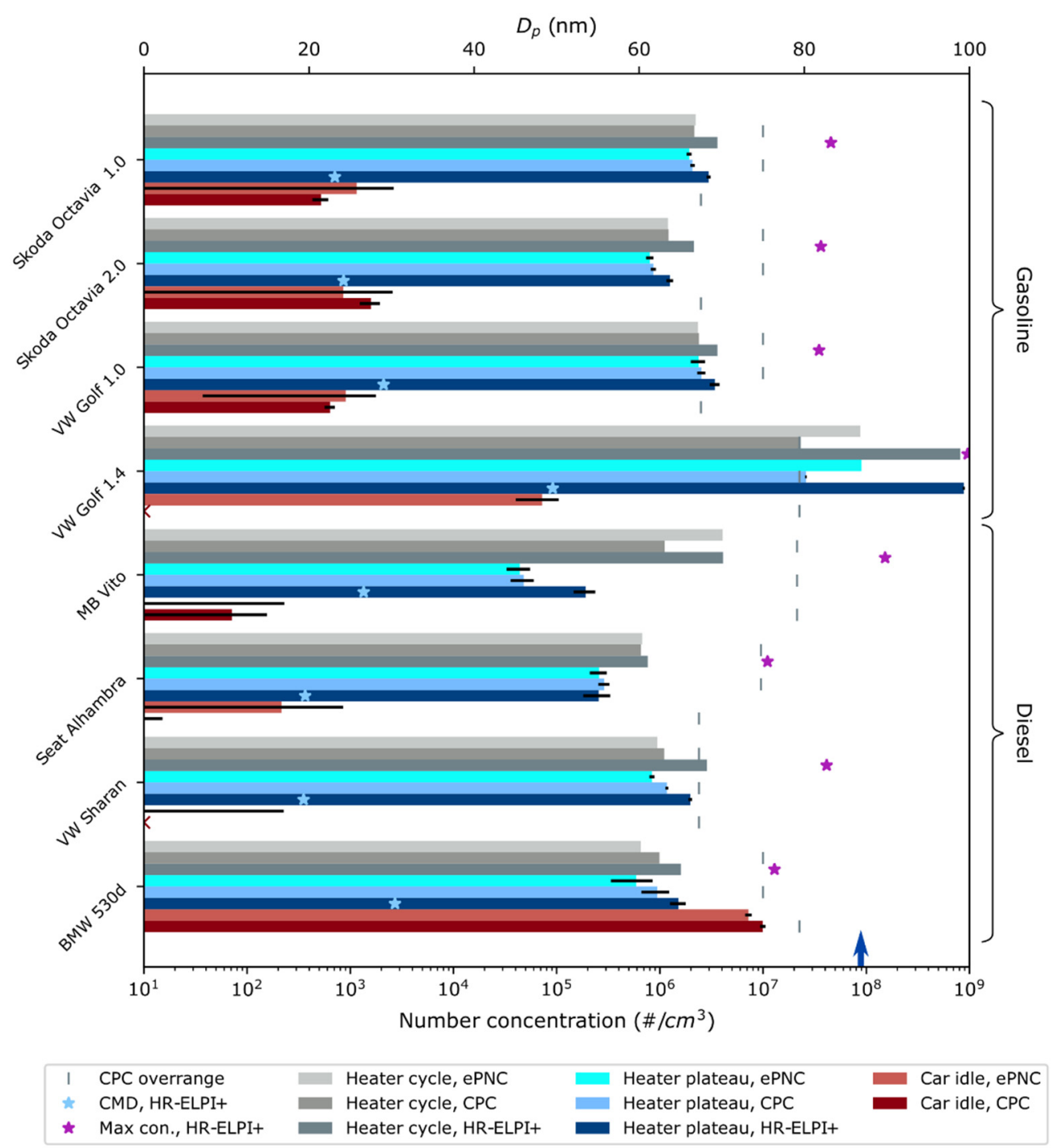

Figure 3. Average particle number concentrations measured from the vehicle exhaust during engine idling and from the auxiliary heater exhaust during its operation. Averages are calculated from both the plateau period as well as from the full range of operation (20 min cycle including the startup and shutdown of the heater). Count median diameters of the particle size distributions measured with the HR-ELPI+ and the CPC overrange limits are presented as well. CPC data were not available for two car idle measurements, these are indicated in the figure with a red " $\mathrm{X}$ ". ePNC overrange limit is indicated with a dark blue arrow.

The differences in heater emissions compared to the emissions from vehicle run comparable time-periods on idle are illustrated in Figure 3. The first thing to note is that emissions from the idle period are drastically lower than for the heater emissions, except for the Euro 3 type BMW 530d. Notably, with the modern DPF-equipped diesel vehicles, the particulate emissions are negligible. The mean particle size in the $\mathrm{AH}$ heater exhaust was typically between 20 and $30 \mathrm{~nm}$ by count median diameter (CMD), which is a smaller particle size than that typically found in the soot mode of combustion engines. However, for one vehicle, we observed a larger mean particle size, although in this case the concentration levels measured from the $\mathrm{AH}$ were over one order of magnitude larger. To give a perspective of temporal variability, the $\mathrm{AH}$ emissions are divided into two parts. The average emissions from the full $20 \mathrm{~min}$ cycle, the heating time recommended by the manufacturer, and from the plateau period are shown in Figure 2. Emissions of the $20 \mathrm{~min}$ cycle were estimated by extending the plateau region of the measurements. This shows 
that the startup and shutdown peaks had a substantial effect on the full cycle emissions, and naturally, they would receive a higher weighting factor if the heating time was shorter.

Figure 3 shows that the exhaust concentration levels of AH units are extremely high, considerably above the values found in the exhaust in general. This observation is based on concentrations, but especially on idle, this translates to direct comparison to particle emissions because the fuel consumption in the AH $(\sim 0.5 \mathrm{~L} / \mathrm{h})$ and idling engine $(\sim 0.7-1.0 \mathrm{~L} / \mathrm{h})$ are relatively similar. One must note that the $\mathrm{AH}$ unit is automatically active under low ambient temperature, and in fact, this assisting heating mode can be the main source of emissions, exceeding the emissions during manual $\mathrm{AH}$ operation pre-ignition. One example is from the tested Seat Alhambra (driven 168,000 km). Assuming a $0.5 \mathrm{~L} / \mathrm{h}$ fuel consumption for the $\mathrm{AH}$ (operated for $1250 \mathrm{~h}$ ), and average fuel consumption of $7 \mathrm{~L} / 100 \mathrm{~km}$ for the vehicle, we estimate that $11,800 \mathrm{~L}$ of fuel $(95.0 \%)$ have been combusted in the engine, whereas $624 \mathrm{~L}$ of fuel $(5.0 \%)$ have been combusted in the AH. This practically implies that if the fuel-based particulate emission factors were over 20 times higher in the $\mathrm{AH}$ compared to the ICE, the total particulate emissions of $\mathrm{AH}$ would exceed that of the ICE. Based on the experiment, we cannot reach this conclusion solely because this measurement does not cover conditions of real driving. However, this study indicates that this may be the case, especially in vehicles that have very efficient filtration systems.

\section{Conclusions}

Our results show that the particle number emissions from auxiliary heaters are about three orders of magnitude higher than from the tailpipes of idling gasoline vehicles. Both gasoline- and diesel-operated AHs displayed the highest concentrations right after the start and the end of combustion, whereas instantaneous concentrations approached nearly steady values during steady operation, of which duration depends on the user preferences. Especially in post-Euro 5b emission standard diesel vehicles that are all equipped with a DPF, AH emissions are highlighted in relation to the very low particle levels found in tailpipes. Results raise the question of whether the use of heaters is justified based on reducing emissions. However, it should be noted that this study does not take a stand on the effect of the $\mathrm{AH}$ on the cold start emissions of the engine or on the reduced engine wear. Thus, more research is needed to characterize the emissions and their environmental impact more thoroughly and give recommendations on the use and further development of heaters.

Author Contributions: P.K., A.A. and S.M. (Santtu Mikkonen). Designed the study; M.N. and A.A. conducted the measurements with the help from P.K., M.O. and S.M. (Sampsa Martikainen); M.N. and A.A. prepared the graphical illustrations; all authors participated in data analysis and manuscript writing. M.N. and A.A. conducted the measurements with the help from P.K., M.O., S.M. (Sampsa Martikainen) and A.R. All authors have read and agreed to the published version of the manuscript.

Funding: This research is part of the "AHMA" project funded by the Jane and Aatos Erkko's Foundation. P.K. acknowledges the Academy of Finland project "EFFi" grant No. 322120 for personal funding. S.M. (Santtu Mikkonen) is supported by the Academy of Finland competitive funding to strengthen university research profiles (PROFI) for the University of Eastern Finland (grant No. 325022). S.M. (Sampsa Martikainen) acknowledges funding from the Kone Foundation. This research has received funding from the Academy of Finland Flagship Programme "ACCC" (Grant numbers 337550 and 337551).

Institutional Review Board Statement: Not applicable.

Informed Consent Statement: Not applicable.

Data Availability Statement: The data will be available upon reasonable request from the corresponding author.

Conflicts of Interest: The authors declare no conflict of interest. 


\section{References}

1. Burnett, R.; Chen, H.; Szyszkowicz, M.; Fann, N.; Hubbell, B.; Pope, C.A.; Apte, J.S.; Brauer, M.; Cohen, A.; Weichenthal, S.; et al. Global estimates of mortality associated with longterm exposure to outdoor fine particulate matter. Proc. Natl. Acad. Sci. USA 2018, 115, 9592-9597. [CrossRef] [PubMed]

2. Ohlwein, S.; Kappeler, R.; Kutlar Joss, M.; Künzli, N.; Hoffmann, B. Health effects of ultrafine particles: A systematic literature review update of epidemiological evidence. Int. J. Public Health 2019, 64, 547-559. [CrossRef] [PubMed]

3. Wihersaari, H.; Pirjola, L.; Karjalainen, P.; Saukko, E.; Kuuluvainen, H.; Kulmala, K.; Keskinen, J.; Rönkkö, T. Particulate emissions of a modern diesel passenger car under laboratory and real-world transient driving conditions. Environ. Pollut. 2020, 265, 114948. [CrossRef] [PubMed]

4. Webasto Group Webasto Facts \& Figures. Available online: https://www.webasto-group.com/en/the-company/facts-figures / (accessed on 10 August 2021).

5. Eberspächer Eberspächer Group Management Report 2020. 2021. Available online: https://www.eberspaecher.com/ presse/pressemitteilungen/einzelansicht/eberspaechers-recent-performance-and-future-prospects-financial-years-2019-2020 (accessed on 10 August 2021).

6. Müller, E.A.; Onder, C.H.; Guzzella, L.; Kneifel, M. Optimal control of a fuel-fired auxiliary heater for an improved passenger vehicle warm-up. Control Eng. Pract. 2009, 17, 664-675. [CrossRef]

7. Högström, R.; Karjalainen, P.; Yli-Ojanperä, J.; Rostedt, A.; Heinonen, M.; Mäkelä, J.M.; Keskinen, J. Study of the PM gas-phase filter artifact using a setup for mixing diesel-like soot and hydrocarbons. Aerosol Sci. Technol. 2012, 46, 1045-1052. [CrossRef]

8. DMIWM. Regulation of the State Secretary for Infrastructure and Water Management of 12 January 2021 No. IENW/BSK2020/125046, Amending the Vehicle Regulation for the Introduction of the PTI Particulate Filter Check with Particle Counter. Ministry of Infrastructure and Water Management. 2021. Available online: https:/ / citainsp.org/wp-content/uploads/2021/03/ Dutch-regulations-for-the-PTI-particle-filter-test-fo-diesel-cars.pdf (accessed on 10 August 2021).

9. Dekati Ltd. Dekati Technologies Web Page. 2021. Available online: https://dekatitechnologies.com/ (accessed on 18 June 2021).

10. Burtscher, H.; Lutz, T.; Mayer, A. A New Periodic Technical Inspection for Particle Emissions of Vehicles. Emiss. Control Sci. Technol. 2019, 5, 279-287. [CrossRef]

11. Arffman, A.; Ukkonen, A.; Nikka, M.; Luntta, E.; Lamminen, E. Method and Apparatus for Monitoring Number Density of Aerosol Particles. Finnish Patent No. FI129009B, 14 May 2021.

12. Järvinen, A.; Aitomaa, M.; Rostedt, A.; Keskinen, J.; Yli-Ojanperä, J. Calibration of the new electrical low pressure impactor (ELPI+). J. Aerosol Sci. 2014, 69, 150-159. [CrossRef] 